\title{
AVALIAÇÃO DE DENTES SUPRANUMERÁRIOS EM EXAMES RADIOGRÁFICOS DE UMA CLÍNICA ODONTOLÓGICA PARTICULAR DO RECÔNCAVO DA BAHIA- UM ESTUDO DE CASOS
}

Roberto Carlos Carvalho Cerqueira ${ }^{1}$, Matheus Augusto Santiago de Melo Conceição ${ }^{1}$, Jéssica Santos da Silva ${ }^{2}$, João Rubens Teixeira de Castro Silva ${ }^{3}$, Larissa Rolim Borges-Paluch ${ }^{4}$

${ }^{1}$ Graduado em Odontologia pela Faculdade Maria Milza (FAMAM), Governador Mangabeira, BA, Brasil.

${ }^{2}$ Graduanda em Odontologia pela FAMAM, Governador Mangabeira, BA, Brasil.

${ }^{3}$ Mestrando em Desenvolvimento Regional e Meio Ambiente da FAMAM, Governador Mangabeira, BA.

${ }^{4}$ Professora Doutora do Mestrado em Desenvolvimento Regional e Meio Ambiente e Biotecnologia da FAMAM, Gov. Mangabeira, BA. E-mail: larissapaluch@gmail.com

Recebido em: 15/08/2020 - Aprovado em: 15/09/2020 - Publicado em: 30/09/2020 DOI: 10.18677/EnciBio_2020C11

\section{RESUMO}

A hiperdontia é o termo referente ao desenvolvimento de dentes supranumerários, os quais são descritos como o excesso de unidades dentárias, ou seja, além da quantidade fisiológica de dentes que constituem as arcadas dentárias. O presente estudo teve como objetivo avaliar a presença de dentes supranumerários e possíveis consequências clínicas associadas em radiografias panorâmicas de pacientes de uma clínica odontológica particular. $O$ estudo foi realizado em uma clínica particular localizada no Recôncavo Baiano sendo utilizadas radiografias panorâmicas de pacientes que realizaram tratamento ortodôntico entre janeiro/2016 a junho/2019. Os dados foram analisados por estatística descritiva (frequência relativa) no Microsoft Office Excel ${ }^{\circledR}$. Do total de 1053 radiografias avaliadas foram observados 26 casos de dentes supranumerários, representando uma prevalência de 2,5\% da população estudada. Dos 26 casos, 14 (54\%) pertenciam a pacientes do sexo masculino e 12 (46\%) ao feminino, ou seja, a anomalia apresentou proporção de, praticamente, 1:1. A maioria dos supranumerários estava localizada na maxila (73\%), numa proporção de 5:1 e sua maior ocorrência foi de forma unilateral (85\%). Com relação à distribuição foram detectados 11 (38\%) casos de parapremolar e 10 $(35 \%)$ de distomolar. Morfologicamente, os dentes rudimentares foram os mais observados, com 15 (56\%) casos. Dentre as consequências clínicas associadas foi observada a impactação de dentes permanentes (21\%), diastema (24\%) e reabsorção radicular de dente adjacente. Dessa forma estudos da ocorrência de dentes supranumerários podem evidenciar como estes se apresentam e auxiliam o cirurgião-dentista na tomada de decisão do tratamento.

PALAVRAS-CHAVE: anomalia dentária. dente impactado. radiografia panorâmica 


\title{
EVALUATION OF SUPERNUMERARY TEETH IN RADIOGRAPHIC EXAMS OF A PRIVATE DENTAL CLINIC IN RECÔNCAVO DA BAHIA
}

\begin{abstract}
Hyperdontia is the term referring to the development of supernumerary teeth, which are described as the excess of dental units, that is, in addition to the physiological quantity of teeth that constitute the dental arches. The present study aimed to evaluate the presence of supernumerary teeth and possible clinical consequences on panoramic radiographs of patients from a private dental clinic. The study was performed in a private clinic located in the Recôncavo Baiano and panoramic radiographs of patients who underwent orthodontic treatment between January/2016 and June/2019 were used. The data were analyzed by descriptive statistics (relative frequency) in Microsoft Office Excel ${ }^{\circledR}$. From a total of 1,053 radiographs evaluated, 26 cases of supernumerary teeth were observed, representing a prevalence of $2.5 \%$ of the studied population. Of the 26 cases, 14 (54\%) belonged to male patients and $12(46 \%)$ to the female, that is, the anomaly presented a ratio of practically $1: 1$. Most supernumeraries were located in the maxilla (73\%), in a ratio of $5: 1$ and their highest occurrence was unilaterally (85\%). Regarding distribution, 11 (38\%) cases of parapremolar and $10(35 \%)$ of distomolar were detected. Morphologically, rudimentary teeth were the most observed, with $15(56 \%)$ cases. Among the associated clinical consequences, the impaction of permanent teeth $(21 \%)$, diastema $(24 \%)$ and root resorption of adjacent tooth. Thus, studies of the occurrence of supernumerary teeth can show how they present themselves and assist the dentist in making treatment decisions.
\end{abstract}

KEYWORDS: dental anomaly. panoramic radiography. impacted tooth.

\section{INTRODUÇÃO}

A espécie humana apresenta um número constante de dentes, cerca de 20 dentes na dentição decídua e 32 dentes na dentição permanente. A presença de supranumerários ou hiperdontia é definida como o excesso de dente na arcada dentária além da quantidade fisiológica, sendo essa uma das anomalias mais recorrente na odontologia (LODI et al., 2017). A mesma pode ser originada por fatores etiológicos diversos, sendo capaz de acarretar distúrbios no desenvolvimento de forma, tamanho e número, por isso é considerada uma anomalia de desenvolvimento (MOURA et al., 2013; STRINGHINI JUNIOR et al., 2015).

Lodi et al. (2017) afirmam que a etiologia do supranumerário não está completamente elucidada na literatura, visto que há algumas teorias que defendem a sua origem. O supranumerário pode ser formado por meio de uma divisão de duas partes iguais, resultando em um dente semelhante ao da arcada normal, e esse processo é chamado de dicotomia do germe dentário. Entretanto, quando acontece esse fenômeno, o dente supranumerário pode se tornar mal formado e cônico.

Os dentes supranumerários podem ocasionar complicações na arcada dentária dos indivíduos que apresentam essa alteração como retardo na erupção, dentes impactados, diastemas, lesões císticas, erupção ectópica, reabsorção radicular dos dentes vizinhos, apinhamento dental, abscesso periodontal e dentes girovertidos. Em casos mais raros, dores de cabeça, parestesia e edema cístico na região pré-maxilar (NUNES et al., 2017).

Ainda de acordo com esses autores os dentes supranumerários são classificados quanto à sua morfologia, sendo denominados de suplementares quando se apresentam semelhantes a um dente normal da maxila e/ou da 
mandíbula e rudimentares quando se mostram numa forma anatômica diferente de um dente normal, podendo apresentar-se na forma conóide (forma de cone), tuberculados (forma de barril), ou na forma de odontomas.

Corteleti et al. (2016) ressaltam que o tratamento pode ser feito com acompanhamento clínico e radiográfico, realização de restauração ou exodontia. No entanto, há várias controvérsias quanto ao período correto para a exodontia desse dente, pois o mesmo pode retardar o processo de erupção do dente permanente, podendo haver a possibilidade de tratamento com reabilitação ortodôntica.

Atualmente o exame mais solicitado para o diagnóstico precoce de alterações dentárias é a radiográfica panorâmica, e possui muitas vantagens como a alta aceitação dos pacientes, emite pouca radiação, permite a visualização de ambas as arcadas e as estruturas adjacentes em uma única imagem e tem baixo custo quando comparado à tomografia (COELHO et al., 2011).

Para o tratamento de dentes supranumerários geralmente são considerados duas possibilidades: a) remoção precoce do elemento dentário, prevenindo futuras complicações, sendo que esta exodontia deve ser realizada cuidadosamente, a fim de evitar danos aos dentes permanentes, como distúrbio de erupção e anquilose; b) sem tratamento cirúrgico, fazendo o monitoramento da erupção dos dentes adjacentes para não causar interferências funcionais e estéticas (MOURA et al., 2013).

Dessa maneira, o presente estudo teve como objetivo geral avaliar a presença de dentes supranumerários e possíveis consequências clínicas associadas em radiografias panorâmicas de pacientes de uma clínica odontológica particular.

\section{MATERIAIS E MÉTODOS}

Tratou-se de um estudo descritivo, documental e quantitativo. O local do estudo foi uma clínica odontológica privada localizada em Santo Antônio de Jesus, no Recôncavo Baiano. Foram utilizadas radiografias panorâmicas do banco de dados digital pertencentes a pacientes que realizaram tratamento ortodôntico na clínica, visto que os pacientes com a anomalia normalmente apresentam consequências clínicas e buscam esse tipo de tratamento a fim de corrigir o problema.

Os critérios de inclusão foram: imagens radiográficas panorâmicas com boa qualidade visual, idade superior a 18 anos, e realização de tratamento ortodôntico no período entre janeiro/2015 a junho/2019. Os critérios de exclusão foram: radiografias de pacientes com histórico de trauma e cirurgia ortognática.

Em relação aos procedimentos e instrumentos de coleta de dados, inicialmente, foi realizada a análise de todas as imagens radiográficas panorâmicas do setor da ortodontia no período estipulado seguindo os critérios de inclusão e exclusão.

A pesquisa ocorreu entre agosto e setembro/2019, sendo que os laudos dos exames de imagem foram separados em dois grupos: sem a anomalia e com a presença de dentes supranumerários. Desse último grupo foi realizada a identificação do sexo, a localização, classificação, forma e as respectivas consequências clínicas do dente supranumerário.

Além disso, foi efetuado estudo minucioso das imagens visando identificar os problemas mais comuns decorrente dos dentes supranumerários, como: retardos na erupção, impacção, diastema, lesões císticas, erupção ectópica, reabsorção radicular dos dentes adjacentes, apinhamento dental, abscesso periodontal. 
O presente projeto possui aprovação do Comitê de Ética em Pesquisa da FAMAM, parecer 3.070.823, CAAE 04216218.4.0000.5025 e atentou para a fidelidade da pesquisa no que diz respeito a todos os princípios éticos contidos na Resolução $n^{\circ}$ 466/12 do Conselho Nacional de Saúde, assegurando confiabilidade, privacidade, anonimato e sigilo da identidade dos sujeitos da pesquisa e, ao mesmo tempo, garantindo imparcialidade, impessoalidade e objetividade na condução do estudo e na redação dos relatórios técnicos.

Os dados foram analisados por meio de estatística descritiva (frequência relativa) no Microsoft Office Excel ${ }^{\circledR}$. Após a coleta de todas as informações foram tabuladas em uma planilha do Microsoft Excel, sendo identificadas as iniciais dos pacientes, sexo, localização do supranumerário, classificação e as respectivas consequências clínicas (diastema, impactação, lesão cística, erupção ectópica, retardo de erupção dos dentes adjacentes, reabsorção radicular, apinhamento e abcesso periodontal).

\section{RESULTADOS E DISCUSSÃO}

Das 1053 radiografias panorâmicas avaliadas foram observados 26 casos de dentes supranumerários, o que representa uma prevalência de $2,5 \%$ da população estudada. Essa prevalência está de acordo com o estudo de Dayube et al. (2011), pois afirmam que a prevalência da anomalia é de $0,5 \%$ a $3,8 \%$ em diferentes populações.

Entretanto, Castro-Silva et al. (2018) realizaram criteriosa análise da distribuição desta anomalia dentária em alguns estados do Brasil, e observaram diferenças na prevalência. Na Região Sul foi avaliado o estado do Paraná com prevalência desta anomalia entre 0,5\% e 2,1\%. Na região Sudeste o Rio de Janeiro apresentou variação entre 1,2\% a 2,3\% e em São Paulo de 1,2\% a 4,7\%. No Centro Oeste o estado do Mato Grosso do Sul apresentou prevalência de 2,1\%, no Pará, localizado na região Norte foi de $2,4 \%$. Foram amostrados três estados na região Nordeste, sendo que no Ceará o valor foi de 1,9\%, na Bahia 3,7\% e 4\% no Piauí.

Torres et al. (2015) analisaram 1054 radiografias panorâmicas de um banco de dados de uma clínica em Teresina-PI e observaram 42 casos de dentes supranumerários, ou seja, uma prevalência de 4,0\% da população estudada. Porém, em estudo de Moura et al. (2013), que analisou 1511 radiografias panorâmicas de pacientes atendidos no Hospital Universitário da Universidade Federal do Piauí (HUUFPI), foram encontrados 30 casos de hiperdontia, o que representa uma prevalência de cerca de apenas 1,99\% da população estudada.

Negrete et al. (2017) avaliaram 1117 radiografias panorâmicas de indivíduos atendidos no curso de pós-graduação em Ortodontia da Universidade Cidade de São Paulo, e observaram 18 radiografias de pacientes com a anomalia, ou seja, uma prevalência de 1,61\%. Já o estudo realizado por Coelho et al. (2011) em Portugal onde foram analisadas 1438 radiografias panorâmicas de pacientes que frequentaram a clínica da Faculdade de Medicina Dentária da Universidade do Porto, observou-se 40 casos de hiperdontia, sendo a prevalência representada por $2,8 \%$.

\section{Sexo}

Das 1053 radiografias analisadas a maioria pertencia a pacientes do sexo feminino, totalizando $632(60 \%)$ pacientes, e dos 26 casos de dentes supranumerários detectados, observou-se semelhança entre os sexos, sendo 12 
(46\%) casos no gênero feminino e 14 (54\%) no masculino, com a prevalência aproximada de 1:1.

Corroborando com a presente pesquisa, Coelho et al. (2011) obtiveram uma proporção de 1:1 em estudo com 1438 radiografias panorâmicas, tal levantamento foi realizado na cidade do Porto, em Portugal. No entanto, os mesmos afirmam não haver diferença estatística entre os sexos, embora a literatura ressalte a maior predominância para o masculino.

Negrete et al. (2017) analisaram 1117 radiografias panorâmicas e registraram 18 casos de supranumerário, tendo $1,61 \%$ de prevalência. Apesar de obterem maior prevalência no sexo feminino $(1,91 \%)$ os autores afirmam que não houve diferença significativa entre os sexos após os testes de Qui-quadrado de Pearson e de Prevalência Binomial, ao nível de $5 \%$. Além disso, relatam que há um consenso na literatura afirmando que o sexo masculino apresenta maior prevalência para essa anomalia. Torres et al. (2015) também observaram uma predominância da anomalia no sexo masculino, na proporção de aproximadamente 2:1. Esses autores acreditam que esses dados podem se alterar em função do tamanho amostral e diferenças geográficas, ressaltando a importância de pesquisas de prevalência em diferentes regiões.

Maciel e Castro-Silva (2016) destacam a hipótese do dimorfismo sexual, visto que têm uma maior ocorrência em homens do que em mulher, numa proporção de $2: 1$. Os mesmos autores também não relatam haver preferência quanto aos grupos étnicos. Moura et al. (2013) evidenciaram que os supranumerários afetaram igualmente ambos os sexos, pois constataram uma proporção de 1:1 em seu estudo. Porém, ressaltam que essa anomalia pode ser decorrente de uma característica peculiar da população estudada e que pode haver relação hereditária.

\section{Localização e Ocorrência na Arcada Dentária}

Quanto à localização dos dentes supranumerários, houve $19(73 \%)$ casos em maxila, quatro (15\%) casos em mandíbula, numa proporção de 7:1. Também foram observados três (12\%) casos em ambas as arcadas. Em relação à ocorrência na arcada dentária foram constatados $22(85 \%)$ casos de dentes supranumerários de forma unilateral e quatro (15\%) bilateral. Corroborando com os dados do presente estudo, Araújo et al. (2018) relataram maior frequência desta anomalia na maxila quando comparada à mandíbula, na proporção de nove a 10 vezes.

Os pesquisadores Teslenco et al. (2017) afirmam que a frequência desse dente é maior na maxila que na mandíbula, numa proporção de 10:1. Para Lara et al. (2013) os dentes supranumerários ocorrem prioritariamente na maxila, embora possam ocorrer em ambas as arcadas.

Entretanto, ao analisar a relação maxila e mandíbula em 1475 radiografias panorâmicas, Dayube et al. (2011), verificaram a ocorrência de 55 indivíduos com a anomalia, sendo que 59\% estavam em maxila e $41 \%$ na mandíbula. Para Moura et al. (2013) a ocorrência da hiperdontia na cavidade oral pode se apresentar tanto de forma unilateral como bilateral, na maxila ou na mandíbula, erupcionados ou impactados, sendo a quantidade de apenas um ou vários dentes supranumerários.

\section{Prevalência e Distribuição dos Dentes Supranumerários}

Dos 26 casos de dentes supranumerários foi observada a seguinte distribuição dos dentes na arcada: cinco (17\%) mesiodente, 11 (38\%) parapremolar, três (10\%) paramolar, 10 (35\%) distomolar. Em relação ao número de dentes houve a ocorrência de um a três dentes a mais na arcada. 
Corroborando o presente estudo, Lara et al. (2013) observaram maior prevalência em parapremolar e acreditam que pode haver associação com a formação dos terceiros molares, os quais evolutivamente, tendem a desaparecer. Logo, obtiveram uma prevalência de $0,45 \%$ em região de pré-molares supranumerários e sempre associados à presença do quarto molar (distomolar), não sendo detectados parapremolar em pacientes com agenesia de terceiros molares.

Os autores ainda ressaltam que esses dados apresentam uma associação estatisticamente significante $(p<0,01)$ entre os parapremolares e os terceiros molares e induzem essa prevalência em grupos específicos de pacientes que têm o distomolar. Dessa forma, os distomolares tiveram uma prevalência de $72,21 \%$ das amostras da população estudada. Entretanto, Negrete et al. (2017) constataram maior ocorrência nos mesiodentes, ou seja, aqueles que estão localizados na parte anterior de maxila e mandíbula, numa proporção de $50 \%$.

Os dados do presente estudo também são confirmados por Coelho et al. (2011), os quais relataram os mesiodentes como maior destaque, representando cerca de $60 \%$, em seu estudo com a população em Porto-PT. Os mesmos ainda afirmam que a prevalência desse dente é de $33 \%$ a $75 \%$ na região estudada. Mas, contrariando a presente pesquisa, Torres et al. (2015) destacaram os distomolares (quartos molares) como dentes mais frequentes, seguidos pelos situados entre os parapremolar (pré-molares inferiores e a região anterossuperior).

\section{Classificação Quanto a Morfologia}

Com relação à morfologia dos dentes supranumerários, foi verificado que dos 26 casos 15 eram de dente rudimentar (56\%) e 12 de suplementar (44\%). Vale ressaltar que foi observado de um a dois dentes classificados como rudimentar e suplementar simultaneamente na mesma radiografia panorâmica.

Segundo Maciel e Castro-Silva (2016), em sua pesquisa, pôde-se constatar maior presença de mesiodentes na forma cônica (rudimentar), sem apresentar semelhança com a anatomia de um dente anterior. Para Araújo et al. (2018), os distomolares (quarto molar) podem ser suplementares eumórficos, mas também podem ser encontrados de forma rudimentar com tamanho reduzido ou com morfologia atípica (conóide, tuberculados ou em forma de odontomas).

Torres et al. (2015) ressaltam que a morfologia dos dentes pode ser anormal, como incisivos apresentando forma de cones e taurodontismo. Logo, ambos iriam trazer danos aos tecidos periodontais e deficiência no crescimento alveolar.

\section{Consequências Clínicas Associadas aos Supranumerários}

Dentre as consequências clínicas associadas mais recorrentes a esta anomalia foi possível observar a presença de 14 (24\%) de ocorrências com diastema, 13 (22\%) episódios de reabsorção radicular de dente adjacente, 12 (21\%) episódios de impactação de dentes permanentes e 11 (19\%) casos de apinhamento dental, 06 (10\%) casos de retardo na erupção de dentes permanentes e $02(4 \%)$ lesões císticas (Gráfico 1). Vale salientar que as consequências clínicas não ocorreram de forma isolada, mas, geralmente estavam associadas, variando de um a quatro alterações concomitantemente na arcada dentária. 
GRÁFICO 1 - Consequências clínicas associadas aos dentes supranumerários de uma clínica particular no recôncavo da Bahia.

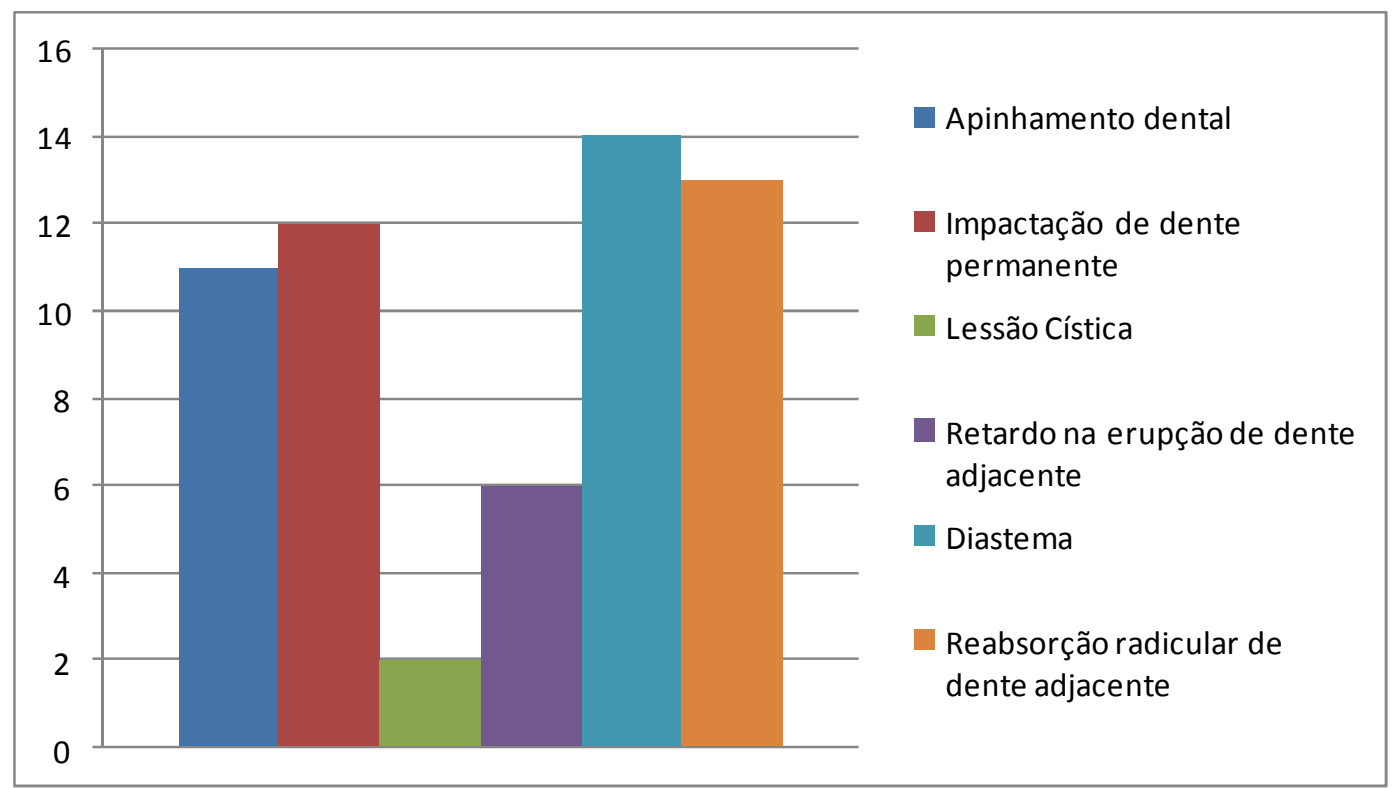

Fonte: autores (2019).

Corroborando o presente estudo, Araújo et al. (2018) explicam que os dentes supranumerários podem causar alterações na arcada dentária, como dente impactado, erupção retardada, más oclusões dentárias, diastemas e reabsorção de dente vizinho. Para Maia et al. (2015) os mesiodentes sempre influenciam na erupção e no alinhamento dos incisivos da maxila, estando relacionados a várias desordens na cavidade oral como: retardo na erupção ou na impacção de dentes adjacentes.

Segundo Maciel e Castro-Silva (2016) os mesiodentes podem contribuir principalmente para mordida aberta, além de retardo na erupção do dente decíduo e permanente, erupção em cavidade nasal, giroversão, formação de cisto dentígero transpasse horizontal aumentado com protrusão de incisivos superiores e selamento labial incompleto. Rocha et al. (2012) ainda ressaltam que as consequências clínicas estão associadas à erupção normal e posição dos dentes adjacentes, podendo haver perda de vitalidade do dente e/ou formação de diastema que pode progredir durante as duas primeiras décadas de vida.

Teslenco et al. (2017) afirmam que em $75 \%$ dos episódios de dentes impactados, os mesmos irão irromper normalmente após a remoção do elemento supranumerário. Porém, há dependência de alguns fatores, como: espaço avaliado para erupção, grau de deslocamento e inclinação do dente não irrompido, tipo de dente supranumerário, tempo decorrido entre o diagnóstico e a intervenção cirúrgica. Por fim, Negrete et al. (2017) chamam atenção para o fato de que a ocorrência de supranumerários é uma condição rara, logo, o mesmo deve ser acompanhado precocemente para que não prejudique o desenvolvimento dos dentes adjacentes.

\section{CONCLUSÕES}

Dos casos de hiperdontia analisados observou-se que as consequências clínicas mais diagnosticadas foram a ocorrência de diastemas e reabsorção radicular de dentes adjacentes. Foi verificado também impactação de dentes permanentes, apinhamentos dentais, retardo na erupção de dente permanente e lesões císticas. 
Destaca-se que o diagnóstico precoce é imprescindível para prevenir problemas dento-alveolares visando o restabelecimento das características estéticas e da oclusão. Para tanto, a radiografia panorâmica apresenta-se como um exame de extrema importância para o diagnóstico preciso e elaboração de um plano de tratamento de sucesso.

Sugere-se a investigação de hiperdontia por regiões no Brasil, destacando a região Nordeste, a fim de verificar e comparar como este fenômeno afeta grupos populacionais. Pois, o conhecimento de tais particularidades pode auxiliar no planejamento das condutas necessárias e o manejo adequado desses indivíduos.

\section{REFERÊNCIAS}

ARAÚJO, L. K.; OLIVEIRA, E. M.; MACIEL, J. A.C.; SAMPIERI, M. B. S.; CASTROSILVA, I. I. Quarto molar inferior e unilateral: caso clínico de anomalia dentária incomum. Brazilian Journal of Surgery and Clinical Research, v. 23, n. 1, p.85-88, 2018.

em: https://www.mastereditora.com.br/periodico/20180606_084541.pdf

CASTRO-SILVA, I. I.; VASCONCELOS, J. L. A.; ALVES, A. D.; BASÍLIO, S. R.; SIEBRA, A. K. A.; MACIEL, J. A. C. Distribuição de anomalias dentárias em cidades do Norte e Nordeste do Brasil. Brazilian Journal Of Surgery And Clinical Research, v. 22, n. 2, p.49-53, 2018. Disponível em: https://www.mastereditora.com.br/periodico/20180405_100842.pdf

COELHO, A.; MACHO, V.; ANDRADE, D.; MACEDO, P.; AREIAS, C Prevalência e distribuição de dentes supranumerários numa população pediátrica: Um estudo radiográfico. Revista Portuguesa de Estomatologia, Medicina Dentária e Cirurgia $\begin{array}{lllll}\text { Maxilofacial, } & \text { v. } 52, \quad \text { n. 4, p.187-193, 2011. DOl: }\end{array}$ https://doi.org/10.1016/j.rpemd.2011.09.005

CORTELETI, J. F.; OTA, C. M.; SARMENTO, L. C.; CHISTÉ, R.; NOVAES, T. F.; IMPARATO, J. C. P. Retenção prolongada de dente decíduo por impactação de dente supranumerário em criança de 12 anos. Revista da Associação Paulista de Cirurgiões Dentistas, v. 70, n. 3, p. 268-272, 2016. Disponível em: http://revodonto.bvsalud.org/scielo.php?pid=S0004-

$52762016000300007 \&$ script=sci_arttext

DAYUBE, A. C.; POMPERMAYER, L.; PENA NETO SEGUNDO, N.; Levantamento das anomalias dentárias de número (supranumerários) em radiografias panorâmicas de um serviço de documentação odontológica da cidade do Salvador Bahia. Revista de Ciências Médicas e Biológicas, v. 10, n. 1, p. 34-38, 2011. DOI: http://dx.doi.org/10.9771/cmbio.v10i1.5248

LARA, T. S.; SILVA, S. B. H. B.; BASTOS, R. T. D. R. M.; OZAWA, T. O.; AYUB, P. V. Prevalência de dentes supranumerários na região dos pré-molares e sua associação com a presença dos terceiros molares. Ortodontia, v. 46, n. 3, p. 262266, 2013.2 Disponível em: https://www.researchgate.net/publication/263855108_Prevalencia_de_dentes_supra numerarios_na_regiao_de_pre-

molares_e_sua_associacao_com_a_presenca_de_terceiros_molares

LODI, C. S.; RODRIGUES, A.; ANGELONI, B. P. Diagnóstico e tratamento de dente supranumerário incluso: relato de caso. Revista Funec Científica-Odontologia, v. 
1, n. $1, \quad$ p. 22-32, 2017. Disponível em: https://www.academia.edu/39535385/diagn\%c3\%93stico_e_tratamento_de_dente_s upranumer\%c3\%81rio_incluso_relato_de_caso

MACIEL, J. A. C.; CASTRO-SILVA, I. I. Mesiodente em paciente odontopediátrico no Ceará, Brasil: relato de caso e revisão de literatura. Brazilian Journal of Surgery and Clinical Research, v. 13, n. 3, p. 38-41, 2016. Disponível em: https://www.researchgate.net/publication/297757463_mesiodente_em_paciente_odo ntopediatrico_no_ceara_brasil_relato_de_caso_e_revisao_de_literatura_mesiodens in_pediatric_dental_patient_in_ceara_brazil_case_report_and_literature_review

MAIA, A. C. D. S. A.; SANTOS, M. O.; SIMÕES, F. X. P. C.; RODRIGUES, S.; NOVAES, T. F.; IMPARATO, J. C. P. Tracionamento de incisivo central superior permanente impactado pela presença de um mesiodente: relato de caso. Revista da Faculdade de Odontologia-UPF, v. 20, n. 1, 2015. DOI: https://doi.org/10.5335/rfo.v20i1.4449

MOURA, W. L. D.; CRAVINHOS, J. C. D. P.; DE MOURA, C. D. V.; FREIRE, S. A. D. S.; MONTEIRO, A. M. O.; PINHEIRO, S. D. A.; RODRIGUES, W. F. B. Prevalência de dentes supranumerários em pacientes atendidos no Hospital Universitário da UFPI: um estudo retrospectivo de cinco anos. Revista de Odontologia da UNESP, v. 42, n. 3, p. 167-171, 2013. DOI: http://dx.doi.org/10.1590/S180725772013000300004

NeGRete, D.; CARVAlHO, P. E. G.; FUZIY, A. A.; TORRES, F. C.; TRIVIÑO, T.; FLAIBAN, E. Prevalência de dentes supranumerários em pacientes de Ortodontia. Revista de Odontologia da Universidade Cidade de São Paulo, v. 27, n. 1, p. 6-13, 2017. DOI: https://doi.org/10.26843/ro_unicid.v27i1.238

NUNES, K. M.; MEDEIROS, M. V.; CERETTA, L. B.; SIMÕES, P. W.; AZAMBUJA, F. G.; SÔNEGO, F. G. F.; PIRES, P. D. S. Dente supranumerário: revisão bibliográfica e relato de caso clínico. Revista de Odontologia da Universidade Cidade de São Paulo, v. 27, n. 1, p. 72-81, 2017. DOI: https://doi.org/10.26843/ro_unicid.v27i1.247

ROCHA, S. DE C. C.; VIDIGAL, B. L.; PEREIRA, A. C.; FONSECA, M. S.; MANZI, F. R. Etiologia, Diagnóstico e Tratamento do Mesiodens - Relato de Caso Clínico Atípico. Arquivo Brasileiro de Odontologia, v. 8, n. 2, p. 49-54, 2012. Disponível em: file:///C:/Downloads/5742-Texto\%20do\%20artigo-22304-1-10-20130909.pdf

STRINGHINI JUNIOR, E.; STANG, B.; OLIVEIRA, L. B. Dentes supranumerários impactados: relato de caso clínico. Revista da Associação Paulista de Cirurgiões Dentistas, v. $\quad 69, \quad$ n. 1, p. 89-94, 2015. Disponível em: http://revodonto.bvsalud.org/scielo.php?pid=S0004-

52762015000100014\&script=sci_arttext

TESLENCO, V. B.; GAETTI-JARDIM, E. C.; DA SILVA, J. C. L. Supranumerários bilaterais em mandíbula: relato de caso. Archives of Health Investigation, v. 3 , n. 6, 2017. DOI: https://doi.org/10.21270/archi.v6i3.1863

TORRES, P. F.; SIMPlíCIO, A. H. D. M.; LUZ, A. R. C. A.; LIMA, M. D. D. M. D.; MOURA, L. D. F. A. D. D.; MOURA, M. S. D. Anomalias dentárias de número em pacientes ortodônticos. Revista de Odontologia da UNESP, v. 44, n. 5, p. 280-284, 2015. DOI: https://doi.org/10.1590/1807-2577.0066 